\title{
Green University Resource Planning on Cloud Computing
}

\author{
Jarumon Nookhong and Prachyanun Nilsook
}

\begin{abstract}
This research is a research and development. It aims to develop Green University Resource Planning on cloud computing. The research is divided into two phases which are 1) document analysis related to Green University indicators, and 2) survey assessment to input data into the Green University Resource Planning System. The research samples are nine experts who are executives and have experience at green universities selected by purposive sampling. The research tool is the survey assessment to input data into the Green University Resource Planning System, which analyses data by considering the mean and standard deviation. According to the document analysis related to Green University Indicators, the research results showed that the categories based on the criteria of UI GreenMetric World University Ranking 2016 suggested that each university has different indicators in relevance to the context, geography, budget, location, internationality, and the survey assessment to input data into the Green University Resource Planning System. The experts found that the overall appropriateness of the survey was rated at the highest level ( $\bar{X}=4.55$, S.D. $=0.69$ ).
\end{abstract}

Index Terms-University resource planning, enterprise resource planning, green university, cloud computing.

\section{INTRODUCTION}

'Green University' refers to a higher education institution, in which a part of the university, or the university as a whole, encourages, manages, and participates in mitigating environmental, economic, social, and health problems arising from resource utilization as much as possible. At the present time, higher education institutions, both in Thailand and elsewhere, have increasingly acknowledged the importance of sustainable and environment-friendly development. Due to the increasing awareness and interest in such development, the author has conceptualized the idea of Green University Resource Planning in higher education institutions based on the idea of Enterprise Resource Planning. The construction of a data structure for higher education helps to save resources and reduce operational processes, as well as effectively deal with problems associated with data processing. As a result, resource allocation processes and related operations are reduced, while management and support services can respond to the teachers' and students' demands more productively. Universities, as is the case with other organizations, are currently being confronted with numerous problems such as resource coordination, budgetary control, personnel in charge of resource allocation, communications, and inter-departmental integration. Hence, University

Manuscript received June 13, 2016; revised December 4, 2016

The authors are with Faculty of Humanities and Social Sciences, Suan Sunandha Rajabhat University, Bangkok (SSRU), Thailand (e-mail: jarumon.no@ssru.ac.th, prachyanunn@kmutnb.ac.th).
Resource Planning begins with the reconstruction of the university's identity. This will lead to a further shift from traditional administration to more effective inter-departmental integration within the university. This is designed to resolve internal problems, integrating information, and improving the overall quality and productivity of the administration that represents the characteristics of each university [1].

The integration of data in terms of the Enterprise Resource Planning System consists of the following sub-modules: Financial, Distribution, Human Resources, and Manufacturing/Production, as are used in business operations, in order to effectively improve operations [2]. University Resource Planning is composed of a variety of modules related to the specific functions of each university including Finance, Human Resources, and Student Project and Activity Management. Moreover, University Resource Planning also involves the use of enormous interactive databases that contain the information about students, personnel, buildings, documents, and financial transactions [3], [4]. In addition, this also includes information about administration, university correspondence, libraries, academic schedules, curriculum, on-campus and off-campus activities, and finance [2]. In the field of research, the higher education system has been modernized; some educational institutions have now become research universities in order to increase their competitiveness on the global stage [5], [6].

In Thailand, the idea of green university development has been extensively used and developed by many higher education institutions. There are 19 universities in Thailand that are qualified to pass the assessment of UI GreenMetric World University Ranking 2016. Chulalongkorn University was ranked the number one green university in Thailand, and the $30^{\text {th }}$ worldwide, followed by Suranaree University of Technology (the $52^{\text {nd }}$ worldwide), Kasetsart University (the $54^{\text {th }}$ worldwide), Mahasarakham University (the $61^{\text {st }}$ worldwide) and Mahidol University (the $71^{\text {st }}$ worldwide), respectively [7], [8]

The ranking criteria used by the UI GreenMetric World University Ranking 2016 [9] are a global standard, indicating a university's efforts in terms of environment-friendly management and sustainable development as they comply with the university's policy. The criteria comprise six aspects set up by Universitas Indonesia in 2011. [10] stated that UI GreenMetric World University Ranking is a framework for each university to inspect its operations in terms of green university development and sustainable development based on the university's policy. Based on the documentary research into green university policy, the criteria can be divided into 10 categories as follows: 1) Waste 2) Resources 3) Ambient/Indoor Air 4) Research 5) Energy 6) Landscaping 7) Transportation by Bus 8) Achieving 
Corporate Social Responsibility 9) Complying with Regulations, and 10) Infrastructure [11].

Based on the aforementioned background, the researcher presents the idea of Green University Resource Planning (GURP) for higher education institutions to become a green university by developing themselves in agreement with the sustainable development and environment-friendly approach, and to develop the Green University Resource Planning System that is practical, measurable, predictable, and supportive to future decision-making.

\section{Purpose of the Research}

To propose green university resource planning on cloud computing.

\section{Methodology}

This research is a research and development project which is divided into two phases as follows:

Phase 1 Document analysis based on Green University Indicators using UI GreenMetric World University Ranking 2016 criteria, a global criteria standard which represents a university's efforts in terms of environment-friendly management and sustainable development as it complies with the university's policy. The author used these criteria as parts of university resource planning for higher education institutions. The criteria comprise six aspects as described below.

1) Setting and Infrastructure: focus on the location, size, and number of green areas within the university.

2) Energy and Climate Change: focus on the efficiency of energy utilization, alternative energy policies, green buildings, and greenhouse gases and electricity usage mitigation policies.

3) Waste: focus on waste management and recycling activities, and paper and plastic usage reduction policies.

4) Water: focus on water conservation and water usage reduction.

5) Transportation: focus on motor vehicle reduction policies, for example, bus and bicycle policy, pedestrian policy, and environment-friendly public transportation policy.

6) Education: focus on courses related to the environment and sustainability, research funds, number of publications, and environment and sustainable development-related websites.

Phase 2 the procedures embedded in the survey assessment to input data into Green University Resource Planning System are identified below:

1) During the first phase, information gained from the Green University Indicators derived from document analysis was used in the survey assessment to input data into the Green University Resource Planning System that was modified according to the context, geography, budget, location and internationality.

2) Proposing the survey assessment to input data into the Green University Resource Planning System to nine experts selected by using purposive sampling for their assessment.

3) Analysing the assessment results according to the assessment criteria by evaluating the mean $(\bar{X})$ and standard deviation (S.D.). The assessment used a 5-level rating scale based on the Likert scale.

\section{RESUltS}

The research results are divided into two parts.

Part 1 Document analysis based on Green University Indicators can be summarized as follows:

1) According to the analysis of the indicators with regard to Setting and Infrastructure, each university accepted the indicators as shown in Table I.

Table I, shows the setting and infrastructure indicators from the UI GreenMetric involving green environment, increased space for green environment, environmental control, and sustainable development. The $50 \%$ or more accepted indicators resulting from the references will be used in the survey assessment to input data into the Green University Resource Planning System in the future.

2) According to the analysis of the indicators under the Energy and Climate Change heading, each university accepted the indicators as shown in Table II.

Table II, illustrates the energy and climate change management indicators from the UI GreenMetric in terms of using energy-saving electronic devices effectively, enforcing alternative energy policies instead of using electricity, green building for energy conservation, and adapting the university to greenhouse gas emission mitigation. The $50 \%$ or more accepted indicators resulting from the references will be used in the survey assessment to input data into the Green University Resource Planning System in the future.

3) According to the analysis of the indicators under the heading of Waste, each university accepted the indicators as shown in Table III.

Table III, presents the waste management indicators from the UI GreenMetric involving waste treatment and recycling, sewerage, and paper and plastic usage reduction policies in a university. The $50 \%$ or more accepted indicators resulting from the references will be used in the survey assessment to input data into the Green University Resource Planning System in the future.

4) According to the analysis of the indicators with regard to Water, each university accepted the indicators as shown in Table IV

Table IV, shows the water management indicators from the UI GreenMetric involving water conservation and decreased water usage. The $50 \%$ or more accepted indicators resulting from the references will be used in the survey assessment to input data into the Green University Resource Planning System in the future.

5) According to the analysis of the indicators under the Transportation heading, each university accepted the indicators as shown in Table $\mathrm{V}$

Table V, illustrates the transportation indicators from the UI GreenMetric involving the transportation policy for reducing the number of vehicles in a university campus; for example, using campus buses and bicycles, walking, and 
using environment-friendly public transportation. The $50 \%$ or more accepted indicators resulting from the references will be used in the survey assessment to input data into the Green University Resource Planning System in the future.
6) According to the analysis of the indicators under the Education heading, each university accepted the indicators as shown in Table VI.

TABLE I: SETTING AND INFRASTRUCTURE

\begin{tabular}{|c|c|c|c|c|c|c|c|c|c|c|c|}
\hline Categories and Indicators & 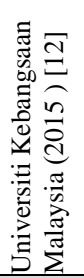 & 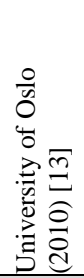 & 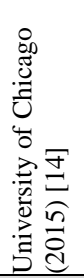 & 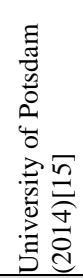 & 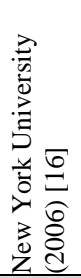 & 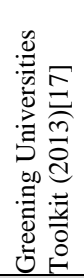 & 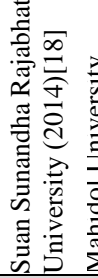 & 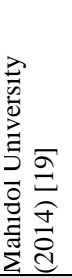 & 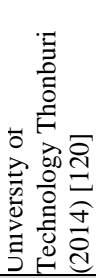 & 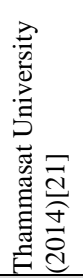 & 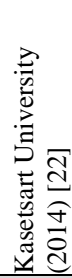 \\
\hline \multicolumn{12}{|l|}{ Setting and Infrastructure } \\
\hline Location & & & & & & & $\checkmark$ & $\checkmark$ & & $\checkmark$ & $\checkmark$ \\
\hline Type of Educational Institution & & & & & & & $\checkmark$ & & & $\checkmark$ & \\
\hline Total Size of Campus (square metres) & & & $\checkmark$ & $\checkmark$ & & & $\checkmark$ & & $\checkmark$ & $\checkmark$ & $\checkmark$ \\
\hline Number of Faculty and Staff & & & & & & & $\checkmark$ & $\checkmark$ & & $\checkmark$ & $\checkmark$ \\
\hline Total Building Areas (square metres) & & & $\checkmark$ & & & $\checkmark$ & $\checkmark$ & & $\checkmark$ & & $\checkmark$ \\
\hline Current Number of Students & & & & & & & $\checkmark$ & & & $\checkmark$ & $\checkmark$ \\
\hline Total Forest Areas (square metres) & & & & $\checkmark$ & $\checkmark$ & & $\checkmark$ & $\checkmark$ & 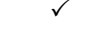 & $\checkmark$ & $\checkmark$ \\
\hline $\begin{array}{l}\text { Total Gardening Areas and Fields (square metres) } \\
\text { Total Green Areas (square metres) }\end{array}$ & & & & $\checkmark$ & $\checkmark$ & $\checkmark$ & $\checkmark$ & $\checkmark$ & $\checkmark$ & $\checkmark$ & $\checkmark$ \\
\hline Budget for Environmental Conservation (baht) & & & & $\checkmark$ & & & $\checkmark$ & $\checkmark$ & & $\checkmark$ & $\checkmark$ \\
\hline
\end{tabular}

TABLE II: SETTING AND INFRASTRUCTURE

\begin{tabular}{|c|c|c|c|c|c|c|c|c|c|c|c|}
\hline Categories and Indicators & 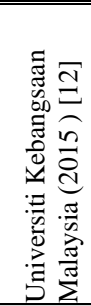 & 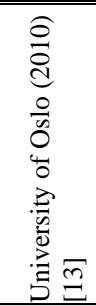 & 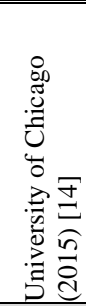 & 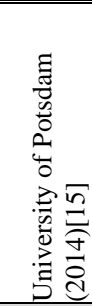 & 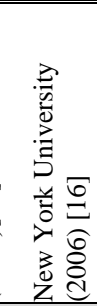 & 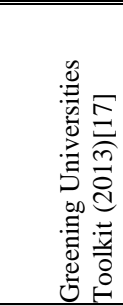 & 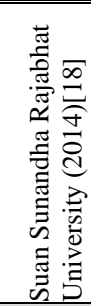 & 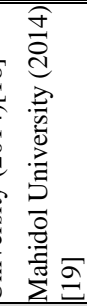 & 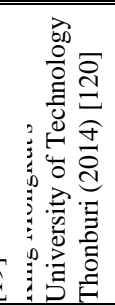 & 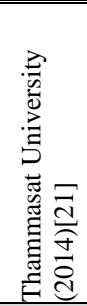 & 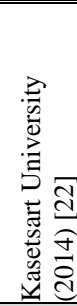 \\
\hline \multicolumn{12}{|l|}{ Energy and Climate Change } \\
\hline Utilization of Energy-Saving Electronic Devices & $\checkmark$ & $\checkmark$ & & & & & $\checkmark$ & $\checkmark$ & $\checkmark$ & $\checkmark$ & $\checkmark$ \\
\hline Alternative Energy Policies & & $\checkmark$ & $\checkmark$ & $\checkmark$ & $\checkmark$ & & $\checkmark$ & $\checkmark$ & $\checkmark$ & $\checkmark$ & $\checkmark$ \\
\hline Amount of Energy Usage (kilowatt/hour per year) & & & $\checkmark$ & $\checkmark$ & $\checkmark$ & $\checkmark$ & $\checkmark$ & $\checkmark$ & $\checkmark$ & $\checkmark$ & $\checkmark$ \\
\hline Energy Conservation Projects & $\checkmark$ & $\checkmark$ & $\checkmark$ & & & & $\checkmark$ & $\checkmark$ & $\checkmark$ & $\checkmark$ & $\checkmark$ \\
\hline Green Building Components & & $\checkmark$ & & & & & $\checkmark$ & $\checkmark$ & $\checkmark$ & $\checkmark$ & $\checkmark$ \\
\hline Global Warming Mitigation Projects & & & & & & & $\checkmark$ & $\checkmark$ & $\checkmark$ & $\checkmark$ & $\checkmark$ \\
\hline Greenhouse Gases Mitigation Projects & $\checkmark$ & $\checkmark$ & $\checkmark$ & $\checkmark$ & & $\checkmark$ & $\checkmark$ & $\checkmark$ & $\checkmark$ & $\checkmark$ & $\checkmark$ \\
\hline \multicolumn{12}{|c|}{ TABLE III: WASTE } \\
\hline Categories and Indicators & 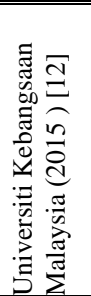 & 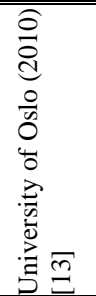 & 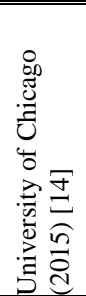 & 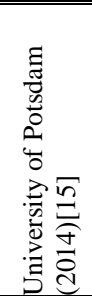 & 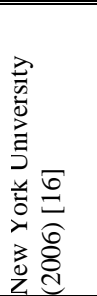 & 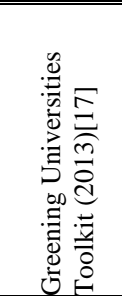 & 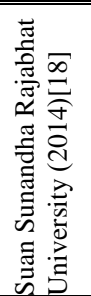 & 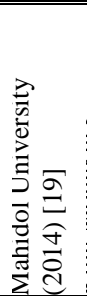 & 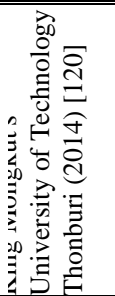 & 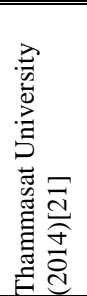 & 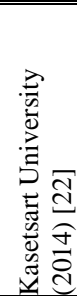 \\
\hline \multicolumn{12}{|l|}{ Waste } \\
\hline Recycling Projects & $\checkmark$ & $\checkmark$ & $\checkmark$ & $\checkmark$ & $\checkmark$ & $\checkmark$ & $\checkmark$ & $\checkmark$ & $\checkmark$ & $\checkmark$ & $\checkmark$ \\
\hline Toxic Waste Recycling & & $\checkmark$ & $\checkmark$ & $\checkmark$ & $\checkmark$ & & $\checkmark$ & $\checkmark$ & $\checkmark$ & $\checkmark$ & $\checkmark$ \\
\hline Organic Waste Disposal & $\checkmark$ & $\checkmark$ & $\checkmark$ & & $\checkmark$ & $\checkmark$ & $\checkmark$ & $\checkmark$ & $\checkmark$ & $\checkmark$ & $\checkmark$ \\
\hline Non-organic Waste Disposal & $\checkmark$ & $\checkmark$ & $\checkmark$ & & $\checkmark$ & & $\checkmark$ & $\checkmark$ & $\checkmark$ & $\checkmark$ & $\checkmark$ \\
\hline Wastewater Treatment & & $\checkmark$ & $\checkmark$ & & $\checkmark$ & & $\checkmark$ & $\checkmark$ & & $\checkmark$ & $\checkmark$ \\
\hline Paper and Plastic Reduction Policies & $\checkmark$ & $\checkmark$ & $\checkmark$ & & $\checkmark$ & & $\checkmark$ & $\checkmark$ & $\checkmark$ & $\checkmark$ & $\checkmark$ \\
\hline
\end{tabular}

Table VI, presents the education indicators from the UI GreenMetric involving the number of courses related to environment and permanence, research funds, the number of academic publications, and the websites about environment and sustainable development. The $50 \%$ or more accepted indicators resulting from the references will be used in the survey assessment to input data into the Green University Resource Planning System in the future.
Therefore, according to the document analysis results based on the Green University Indicators summarized from the indicator analysis into six parts and based on the criteria of the UI GreenMetric World University Ranking 2016, each indicator was differentiated by the context, geography, budget, location and internationality. The $50 \%$ or more accepted indicators resulting from the references will be used in the survey assessment to input data into the Green 
University Resource Planning System in the future [23]-[25]. summarized as Table VII.

Part 2 the survey assessment to input data into the Green

University Resource Planning System by the experts can be

TABLE IV: WATER

\begin{tabular}{|c|c|c|c|c|c|c|c|c|c|c|c|}
\hline Categories and Indicators & 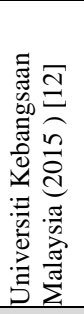 & 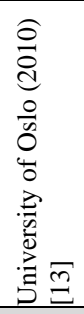 & 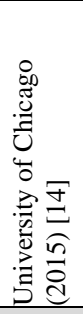 & 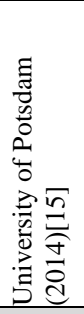 & 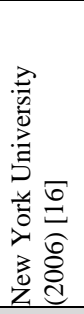 & 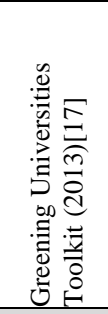 & 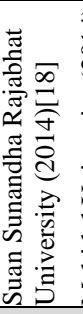 & 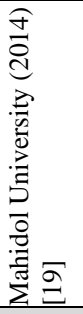 & 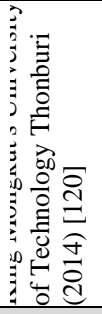 & 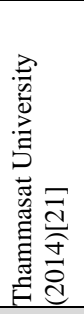 & 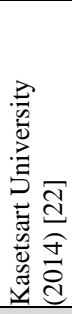 \\
\hline \multicolumn{12}{|l|}{ Water } \\
\hline $\begin{array}{l}\text { Water Conservation Projects } \\
\text { The Percentage of Water Usage }\end{array}$ & $\begin{array}{l}\checkmark \\
\checkmark \\
\end{array}$ & $\checkmark$ & $\checkmark$ & $\checkmark$ & $\checkmark$ & $\checkmark$ & $\checkmark$ & $\begin{array}{l}\checkmark \\
\checkmark \\
\end{array}$ & $\checkmark$ & $\checkmark$ & $\checkmark$ \\
\hline
\end{tabular}

TABLE V: TRANSPORTATION

\begin{tabular}{|c|c|c|c|c|c|c|c|c|c|c|c|}
\hline Categories and Indicators & 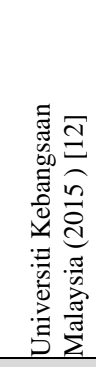 & 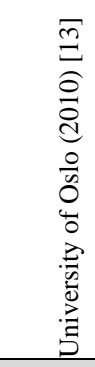 & 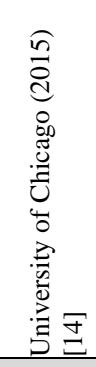 & 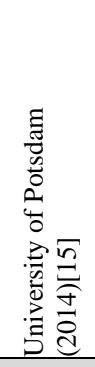 & 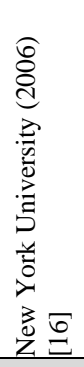 & 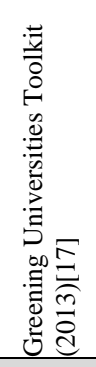 & 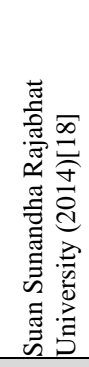 & 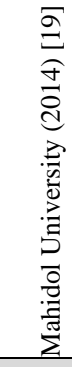 & 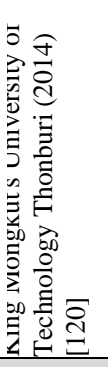 & 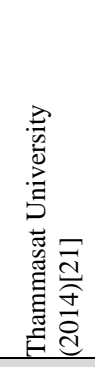 & 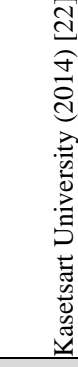 \\
\hline \multicolumn{12}{|l|}{ Transportation } \\
\hline Number of Vehicles on Campus & & & $\checkmark$ & & & & $\checkmark$ & $\checkmark$ & & $\checkmark$ & $\checkmark$ \\
\hline Average Number of Cars on Campus per Day & & & $\checkmark$ & & & & $\checkmark$ & $\checkmark$ & & $\checkmark$ & $\checkmark$ \\
\hline Average Number of Bicycles on Campus per Day & & & $\checkmark$ & & & & $\checkmark$ & $\checkmark$ & & $\checkmark$ & $\checkmark$ \\
\hline $\begin{array}{l}\text { Cars and Motorcycles Reduction Policy on } \\
\text { Campus }\end{array}$ & & $\checkmark$ & $\checkmark$ & & & & $\checkmark$ & $\checkmark$ & $\checkmark$ & $\checkmark$ & $\checkmark$ \\
\hline Parking Restriction and Reduction Policies & & $\checkmark$ & $\checkmark$ & & & & $\checkmark$ & $\checkmark$ & $\checkmark$ & $\checkmark$ & $\checkmark$ \\
\hline Campus Buses & & $\checkmark$ & $\checkmark$ & & $\checkmark$ & & $\checkmark$ & $\checkmark$ & $\checkmark$ & $\checkmark$ & $\checkmark$ \\
\hline Pedestrians and Bicycles Facilitation Policies & & $\checkmark$ & $\checkmark$ & & $\checkmark$ & & $\checkmark$ & $\checkmark$ & $\checkmark$ & $\checkmark$ & $\checkmark$ \\
\hline \multicolumn{12}{|c|}{ TABLE VI: EDUCATION } \\
\hline Categories and Indicators & 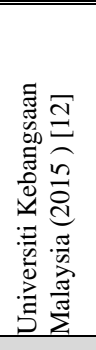 & 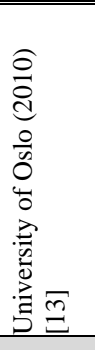 & 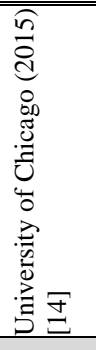 & 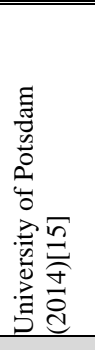 & 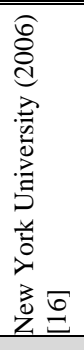 & 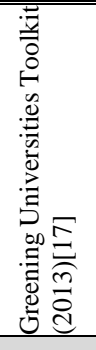 & 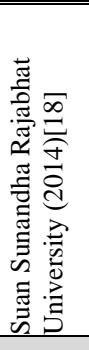 & 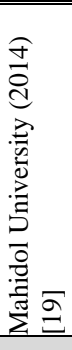 & 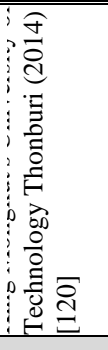 & 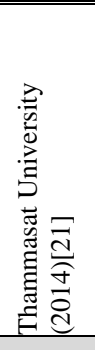 & 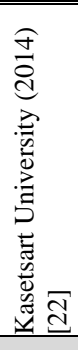 \\
\hline \multicolumn{12}{|l|}{ Education } \\
\hline $\begin{array}{l}\text { Total Number of Environment-related Courses } \\
\text { and Disciplines }\end{array}$ & & & & & $\checkmark$ & & $\checkmark$ & $\checkmark$ & $\checkmark$ & $\checkmark$ & $\checkmark$ \\
\hline Total Number of All Courses and Disciplines & & & & & & & $\checkmark$ & $\checkmark$ & & $\checkmark$ & $\checkmark$ \\
\hline $\begin{array}{l}\text { Total Number of Environment-related Research } \\
\text { Funds }\end{array}$ & & $\checkmark$ & & & & & $\checkmark$ & $\checkmark$ & $\checkmark$ & $\checkmark$ & $\checkmark$ \\
\hline Total Number of Research Funds & & $\checkmark$ & & & & & $\checkmark$ & $\checkmark$ & & $\checkmark$ & $\checkmark$ \\
\hline $\begin{array}{l}\text { Total Number of Environment-related } \\
\text { Publications }\end{array}$ & & & $\checkmark$ & & & & $\checkmark$ & $\checkmark$ & $\checkmark$ & $\checkmark$ & $\checkmark$ \\
\hline Total Number of Publications & & & & & & & $\checkmark$ & $\checkmark$ & & $\checkmark$ & $\checkmark$ \\
\hline $\begin{array}{l}\text { Total Number of Environment-related Student } \\
\text { Organizations }\end{array}$ & & $\checkmark$ & & & & & $\checkmark$ & $\checkmark$ & $\checkmark$ & $\checkmark$ & $\checkmark$ \\
\hline $\begin{array}{l}\text { Total Number of Environment-related } \\
\text { Informative Websites } \\
\end{array}$ & & $\checkmark$ & $\checkmark$ & & & & $\checkmark$ & $\checkmark$ & $\checkmark$ & $\checkmark$ & $\checkmark$ \\
\hline
\end{tabular}




\begin{tabular}{|c|c|c|c|}
\hline Assessment Criteria & $\bar{X}$ & S.D. & Results \\
\hline \multicolumn{4}{|l|}{ Setting and Infrastructure } \\
\hline Total Size of Campus (square metres) & 4.56 & 0.68 & Highest \\
\hline Total Building Area (square metres) & 4.44 & 0.68 & High \\
\hline Total Forest Areas (square metres) & 4.44 & 0.68 & High \\
\hline Total Gardening Areas and Fields (square metres) & 4.56 & 0.68 & Highest \\
\hline Total Absorbed Water Areas (square metres) & 4.44 & 0.68 & High \\
\hline $\begin{array}{l}\text { Current Number of Professors, Staff and Students } \\
\text { (person/academic year) }\end{array}$ & 4.56 & 0.68 & Highest \\
\hline $\begin{array}{l}\text { Budget for Environmental Conservation (total budget of } \\
\text { university) }\end{array}$ & 4.56 & 0.96 & Highest \\
\hline Total & 4.51 & 0.72 & Highest \\
\hline \multicolumn{4}{|l|}{ Energy and Climate Change } \\
\hline Utilization of Energy-Saving Electronic Devices & 4.78 & 0.63 & Highest \\
\hline Alternative Energy Policies & 4.78 & 0.63 & Highest \\
\hline Amount of Energy Usage (kilowatt/hour per year) & 4.67 & 0.67 & Highest \\
\hline Energy Conservation Projects & 4.78 & 0.63 & Highest \\
\hline Green Building Components & 4.67 & 0.47 & Highest \\
\hline Greenhouse Gas Mitigation Projects & 4.78 & 0.63 & Highest \\
\hline Total & 4.74 & 0.61 & Highest \\
\hline \multicolumn{4}{|l|}{ Waste } \\
\hline Recycling Projects & 4.89 & 0.31 & Highest \\
\hline Toxic Waste Recycling & 4.56 & 0.96 & Highest \\
\hline Organic Waste Disposal & 4.78 & 0.63 & Highest \\
\hline Inorganic Waste Disposal & 4.67 & 0.67 & Highest \\
\hline Wastewater Treatment & 4.78 & 0.63 & Highest \\
\hline Paper and Plastic Reduction Policies & 4.89 & 0.31 & Highest \\
\hline Total & 4.76 & 0.58 & Highest \\
\hline \multicolumn{4}{|l|}{ Water } \\
\hline Water Conservation Project & 4.78 & 0.42 & Highest \\
\hline The Percentage of Water Usage (cubic meter/academic & 4.78 & 0.63 & Highest \\
\hline Total & 4.78 & 0.52 & Highest \\
\hline \multicolumn{4}{|l|}{ Transportation } \\
\hline Number of Vehicles on Campus & 4.22 & 0.79 & High \\
\hline $\begin{array}{l}\text { Amount of Gasoline Usage of a Vehicle (litre/academic } \\
\text { year) }\end{array}$ & 4.56 & 0.50 & Highest \\
\hline Number of Cars on Campus Per Capita (car/person) & 4.44 & 0.68 & High \\
\hline $\begin{array}{l}\text { Campus Buses (a bus for travelling inside college or } \\
\text { university both of free or having a service charge) }\end{array}$ & 4.56 & 0.68 & Highest \\
\hline Limited Vehicle Usage on Campus Policies & 4.33 & 0.82 & High \\
\hline Parking Restriction and Reduction Policies & 4.33 & 0.82 & High \\
\hline $\begin{array}{l}\text { Promoting Bicycle Usage Instead of Cars on Campus } \\
\text { Policies }\end{array}$ & 4.56 & 0.68 & Highest \\
\hline Pedestrians and Bicycle Facilitation Policies & 4.56 & 0.68 & Highest \\
\hline Total & 4.44 & 0.71 & High \\
\hline \multicolumn{4}{|l|}{ Education } \\
\hline $\begin{array}{l}\text { Total Number of All Courses and Disciplines (per } \\
\text { academic year) }\end{array}$ & 4.22 & 0.92 & High \\
\hline $\begin{array}{l}\text { Total Number of Environment-related Courses and } \\
\text { Disciplines (per total amount of courses and disciplines } \\
\text { which are opened) }\end{array}$ & 4.56 & 0.50 & Highest \\
\hline Total Number of Research Funds (per academic year) & 4.00 & 0.94 & High \\
\hline $\begin{array}{l}\text { Total Number of Environment-related Research } \\
\text { Funds (per total research budget) }\end{array}$ & 4.44 & 0.68 & High \\
\hline $\begin{array}{l}\text { Total Number of Environment-related Publications Per } \\
\text { Academic Year }\end{array}$ & 4.44 & 0.96 & High \\
\hline Total Number of Publications Per Academic Year & 4.56 & 0.68 & Highest \\
\hline $\begin{array}{l}\text { Total Number of Environment-related Student } \\
\text { Organizations }\end{array}$ & 4.33 & 0.82 & High \\
\hline $\begin{array}{l}\text { Total Number of Environment-related Informative } \\
\text { Websites }\end{array}$ & 4.33 & 0.82 & High \\
\hline Total & 4.36 & 0.79 & High \\
\hline
\end{tabular}

As shown in Table VII, the results of the survey assessment to input data into the Green University Resource Planning System by the experts indicates the overall appropriateness of the survey was rated at the highest level $(\bar{X}=4.55$, S.D. $=0.69)$. Moreover, the survey can be summarized as follows: 1) Setting and Infrastructure criteria: the appropriateness of the survey was rated at the highest level $(\bar{X}=4.51$, S.D. $=0.72)$. 2) Energy and Climate Change criteria: the appropriateness of the survey was rated at the highest level $(\overline{\mathrm{X}}=4.74$, S.D. $=0.61)$. 3) Waste criteria: the appropriateness of the survey was rated at the highest level $(\bar{X}=4.76$, S.D. $=0.58)$. 4) Water criteria: the appropriateness of the survey was rated at the highest level ( $\bar{X}=4.78$, S.D. $=0.52$ ). 5) Transportation criteria: the appropriateness of the survey was rated at a high level ( $\bar{X}$ $=4.44$, S.D. $=0.71) .6$ ) Education criteria: the appropriateness of the survey was rated at a high level $(\overline{\mathrm{X}}=4.36$, S.D. $=0.79)$. 


\section{DISCUSSION}

The results of the research on the idea of Green University Resource Planning on cloud computing, analysing the indicators in the criteria of the UI GreenMetric World University Ranking 2016, were assessed into six areas consisting of 1) setting and infrastructure, 2) energy and climate change, 3) waste, 4) water, 5) transportation, and 6) education. The $50 \%$ or more accepted indicators resulting from the references will be used in the survey assessment to input data into the Green University Resource Planning System. Therefore, the researcher used the data derived from synthesizing to support the input of data into the Green University Resource Planning System specifically related to the university operations [2], [26], [27]. This was differentiated by the context, geography, budget, location and internationality observed in the university environment to develop the organization sustainably and in an environment-friendly way [28]-[30].

\section{ACKNOWLEDGMENT}

This research received a partial thesis research grant for graduate students from the Graduate College at King Mongkut's University of Technology North Bangkok. The researcher would like to express sincere gratitude to the Vacation Education Technology Research Center of the Science and Technology Research Institute, King Mongkut's University of Technology North Bangkok, for supporting this research.

\section{REFERENCES}

[1] M. Li et al., "Research on digital campus of higher colleges and its management platform," presented at 2010 International Coriference on Educational and lriformation Technology (ICEIT 2010).

[2] A. Mudaliar, V. Garde, and D. Sharma, "Educational resource planning - A framework for educational institutions," presented at Second International Conference on Emerging Trends in Engineering and Technology (ICETET-09), 2009.

[3] N. Pollock and J. Cornford, "ERP systems and the university as a unique organisation," Information Technology \& People, 2004.

[4] H. Abdellatif, "ERP in higher education: A deeper look on developing countries," IEEE, 2014

[5] P. Nilsook and T. Sriwongkol, "The development of multi-weblog with knowledge management for Thailand's higher education," presented at 2009 International Conference on Information and Multimedia Technology, 2009.

[6] P. Nilsook and P. Wannapiroon, "International distance consulting via web conferencing," presented at 2014 International Journal of Emerging Technologies in Learning (IJET), 2014.

[7] Office of the Education Council, The Study of Educational Statistics and Indicators, Statistical and Indicator Analysis, and Education Ranking for Increasing Competitiveness, vol. 9, pp. 1-8, February 2014.

[8] Overall Ranking. (2015). [Online]. Available: http://greenmetric.ui.ac.id/overall-ranking-2015/

[9] Guidelines of UI GreenMetric Ranking Team Ver 1.12 September 16th 2016.

[10] Y. Wang et al., "Moving towards an ecologically sound society? Starting from green universities and environmental higher education," SciVerse ScienceDirect International Journal of Cleaner Production, 2013.

[11] J. Nookhong and P. Nilsook, "The analysis of green university resource planning on cloud computing," presented at the sixth International e-Learning Conference 2015 (IEC2015).

[12] L. Mamat et al., "Determining the level of environmental sustainability practices at campus dormitories using green indicators," Asian Social Science Canadian Center of Science and Education, 2015.
[13] M. Faghihimani, Maryam Best Green University Practice Version: Steering Group.

[14] Sustainability at the University of Chicago. (2015). Metrics and Indicators. [Online]. Available: http://sustainability.uchicago.edu/ssp/metrics/\#High-Performance Buildings

[15] T. Lipp, T. Gäbler, and S. Dirlich. (2014). Re-green system of indicators. [Online]. Available: http://www.regreen.eu/uploads/cms/20140808150216_Expertise_final _version_June_2014.pdf.

[16] Greening the Urban Campus. A sustainability assessment of New York University. [Online]. Available: https://www.nyu.edu/sustainability/pdf/gallatinassessment.pdf

[17] Greening Universities Toolkit. [Online]. Available: http:// www.unep.org/training/docs/Greening_Universities_Toolkit.pdf

[18] The Draft of Suan Sunandha Rajabhat University's Promotion Criteria of Green University Ranking by UI Greenmetric World University Ranking.

[19] Mahidol Sustainable University. [Online]. Available: http://www.mahidol.ac.th/green/

[20] Sustainability at KMUTT. [Online]. Available: http://sustainable.kmutt.ac.th/

[21] Thammasat University. Sustainable University. [Online]. Available: http://sustainable.tu.ac.th/homehtml/h.html

[22] Kasetsart University. (2014). Green Campuses. [Online]. Available: http://greencampus.ku.ac.th/

[23] P. Mell and T. Grance, "The NIST definition of cloud computing: Recommendations of the National Institute of Standards and Technology," NIST Special Publication800-145.

[24] C. G. Gobinda, Content in the Cloud: Towards a Green Information Service Model.

[25] N. Sultan, "Cloud computing for education: A new dawn?" International Journal of Information Management, 2010.

[26] S. Pumchalerm, P. Nilsook, and N. Jeerungsuwan, "Intelligent cooperative education process management model on cloud computing technology for higher education institutes in Thailand," International Journal of Information and Education Technology, vol. 6, no. 10, October 2016, pp. 791-794.

[27] T. Rodmunkong, P. Wannapiroon, and P. Nilsook, International Journal of Signal Processing Systems, vol. 2, no. 2, December 2014, pp. 160-165.

[28] I. Rodtusana, Green University. HCU Journal, vol 18, no 36, pp. 171-188.

[29] P. Lertchaiprasert and P. Wannapiroon, "Study of e-waste management with green ICT in Thai higher education institutions," IJEEEE, 2013 vol. 3, no. 3, pp. 239-243.

[30] P. Lertchaiprasert, P. Wannapiroon, and P. Nilsook, "The confirmatory factors analysis of e-waste management model with green ICT," presented at the Sixth International e-Learning Conference, pp. 87-92.

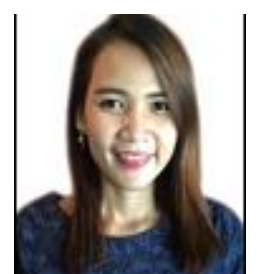

Jarumon Nookhong is lecturer of information science, Faculty of Humanities and Social Sciences at Suan Sunandha Rajabhat University, Bangkok (SSRU), Thailand. She got her bachelor degree of information science (information management) Walailak University. Also, she graduated her master of sciences (management information system) King Mongkut's University of Technology North Bangkok and the Ph.D. candidate in information and communication technology for education from King Mongkut's University of Technology North Bangkok, Thailand. She's research focuses on Data mining, knowledge management, artificial neural networks, green ICT, cloud computing and green cloud system.

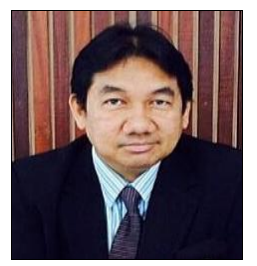

Prachyanun Nilsook is with the Faculty of Technical Education, King Mongkut's University of Technology North Bangkok (KMUTNB), Bangkok, Thailand. 